\title{
The immunohistochemical analysis of membrane-bound CD55, CD59 and fluid-phase FH and FH-like complement inhibitors in cancers of ovary and corpus uteri origin
}

\author{
LUCYNA KAPKA-SKRZYPCZAK ${ }^{1,2}$, EWA WOLINSKA ${ }^{3}$, GRZEGORZ SZPARECKI ${ }^{3}$, \\ MAGDALENA CZAJKA ${ }^{2}$, MACIEJ SKRZYPCZAK ${ }^{4}$
}

'Department of Medical Biology and Translational Research, University of Information Technology and Management, Faculty of Medicine, Rzeszow, Poland

${ }^{2}$ Department of Molecular Biology and Translational Research, Institute of Rural Health, Lublin, Poland

${ }^{3}$ Department of Pathology, Medical University of Warsaw, Warsaw, Poland

${ }^{4}$ Second Department of Gynecology, Medical University of Lublin, Lublin, Poland

\begin{abstract}
One of the potential therapeutic methods of cancer treatment is the immunotherapy with monoclonal antibodies. This kind of therapy, although devoid of serious side effects, has often insufficient efficacy. The presence of complement inhibitors on the cancer cells, which are able to inactivate complement-mediated immune response represents one of the main reasons for the inefficiency of such therapy. In our studies we investigated the expression of main membrane-bound and fluid-phase complement regulators: CD55, CD59 and factor H/factor H-like in tumour samples of ovarian and corpus uteri cancer. Tissue samples were collected from 50 patients and stained immunohistochemically, with the use of peroxidase-based immunodetection system. Immunohistochemical analysis revealed that complement inhibitors are present in examined tumors although their presence is heterogenous. The most prevalent is the presence of factor $\mathrm{H} / \mathrm{H}$-like, localized mostly in tumor stroma and within vascular structures. Membrane bound complement inhibitors are less prominently expressed by cancer cells. CD55 was detected in low percentage of cells, predominantly within cancer tubules. CD59 immunoreactivity was more prevalent in cancer cells, and was localized particularly at the margin of cancer cell tubules. Our results demonstrate that the most prominent complement inhibitor in cancer of ovary and corpus uteri origin is factor H/factor H-like. Blocking or downregulation of this inhibitor should be taken into consideration with regards to improving the efficiency of immunotherapy with monoclonal antibodies.
\end{abstract}

Key words: factor H, CD55, CD59, gynecological cancer.

(Cent Eur J Immunol 2015; 40 (3): 349-353)

\section{Introduction}

Ovarian cancer constitutes a global disease affecting approximately 225,500 women per year and cancer of corpus uteri origin approximately 287,100 [1]. Its mortality remains high due to inefficient therapy and the advanced stage at diagnosis. Although $80 \%$ of affected women benefit from the first-line therapy, tumour recurrence occurs in almost all these patients at a median of 15 months after diagnosis. Similarly it is estimated that cancer of corpus uteri origin affects approximately 287,100 women per year [1]. Therefore, there is a growing need to develop new therapies in these indications. One promising approach for cancer therapy seems to be an immunotherapy with monoclonal antibodies directed specifically against cancer cells. This technique applies natural capabilities of the immunological system to eliminate cancer cells with complement-dependent cytotoxicity and antibody-dependent cellular cytotoxicity. This kind of therapy was found to be effective in some types of malignancies, i.e. in lymphoma, where a high

Correspondence: Ewa Wolinska, Department of Pathology, Medical University of Warsaw, Zwirki and Wigury 61, 02-106 Warsaw, Poland, e-mail: ewa.wilczek@wum.edu.pl 
curative rate was achieved [2]. Though immunotherapy has shown complete remission in some patients, in others the effect is insignificant. The underlying mechanism of this discrepancy is unknown. As was established for a number of solid tumors, one important obstacle to this type of treatment is the presence of complement inhibitors on the surface of cancer cells or fluid-phase inhibitors localized within the tumor stroma. These proteins facilitate escape of tumor cells from the complement mediated injury. The most important membranous complement inhibitors are CD55 (decay accelerating protein, DAF) and CD59 (protectin). CD55 is a glycosylphosphatidylinositol (GPI) - anchored protein found on the surface of epithelial, endothelial and hematopoietic cells [3, 4]. CD55 regulates the complement activation cascade by accelerating the decay of intermediate products of the activation, convertases C3 and C5 [5]. As numerous studies showed, CD55 is overexpressed in many types of cancer, localized both on the surface and within tumor microenvironment [6, 7]. CD59 (also known as protectin), similarly to CD55, is a GPI-anchored glycoprotein widely distributed on the cellular membrane of erythrocytes, platelets, lymphocytes and endothelial cells [8]. Protectin acts at the terminal stages of the complement activation by preventing the formation of the membrane attacking complex (MAC) on cell surfaces [9, 10]. CD59 expression was broadly examined in different types of cancer, however results of these studies are not unequivocal [11-13].

Another important group of complement regulators are those localized in body fluids. The pivotal role within this group is played by the soluble factor $\mathrm{H}$. This huge $(150 \mathrm{kDa})$ glycoprotein is present in serum in the $400-500$ $\mu \mathrm{g} / \mathrm{ml}$ concentration [14]. It possess 20 domains, called SCR (short consensus repeats), which can be modified by post-translation mechanisms. Factor $\mathrm{H}$ inhibits an alternative pathway of the complement system mainly at the level of $\mathrm{C} 3$ convertase formation. It has the ability to recognize sialic acid residues on cellular surfaces, thus can distinguish self versus pathogenic cells [15]. Within this family of proteins there is also factor $\mathrm{H}$-like, alternatively spliced form of the factor $H$ gene [16]. This shorter version of factor $\mathrm{H}$ shares very high homology with $\mathrm{FH}$ and may act as a cofactor for $\mathrm{C} 3 \mathrm{~b}$ degradation and as an accelerator of $\mathrm{C} 3$ and $\mathrm{C} 5$ decay [17]. Factor $\mathrm{H}$ and factor H-like, as was shown by recent studies, are expressed at high levels by cancer cells. Its presence on the cell surface can markedly reduce complement mediated cytotoxicity [18]. In the present study we aimed to investigate the expression of both membrane bound- CD55, CD59 and fluid-phase factor $\mathrm{H}$ expression in ovarian and corpus uteri cancer tissues. Furthermore, we attempted to investigate the correlation between the expression level of these complement inhibitors and clinical and histopathological characteristics of the tumour. Advanced understanding of molecular pathogenesis of cancer types examined may have the potential impact on the development of novel targeted therapies.

\section{Material and methods}

Immunohistochemical analysis was performed on 45 formalin fixed paraffin embedded tumour specimens and 5 control specimens from non-malignant tissue. The detection of complement inhibitors was performed by anti-CD55 and anti-CD59 mouse monoclonal antibodies (Serotec, UK clone MCA1614 at a concentration of 50 $\mathrm{mg} / \mathrm{ml}$ and MCA1054 at a concentration of $20 \mathrm{mg} / \mathrm{ml}$, respectively). For the factor $\mathrm{H}$ immunostaining analysis, a goat polyclonal antibody was used (Quidel, USA). In brief, the staining procedure was as follows: deparaffinization and rehydration of sections, antigen retrieval done in the high temperature boiling in the phosphate buffer of pH 8.0 (Target Retrieval Solution pH 8.0, DakoCytomation, Denmark), blocking of endogenous peroxidase by the incubation in $3 \%$ hydrogen peroxide, blocking of non-specific binding sites by the incubation in 5\% normal donkey serum (Jackson Immunoresearch, USA) and antibody application. For the primary antibodies detection, horse anti-mouse ImmPress Detection System (Vector Laboratories, USA) and donkey-anti-goat polyclonal antibody-HRP conjugated (Jackson Immunoresearch) were used. The reaction was carried out by 3,3'-diaminobenzidine used as a chromogen (DakoCytomation). The immunoreactivity analysis included counting of immunopositive cells in 10 high power fields (HPF) - in the case of CD55 and CD59 and morphometric analysis (measuring both the area covered by the immunoreactivity and the intensity of reaction) of 10 representative images in the case of factor H. Results were presented as an average of obtained figures.

\section{Results}

On the basis of performed experiments we found that in both ovarian and corpus uteri cancer sections, the fluid-phase complement inhibitor factor $\mathrm{H} /$ factor $\mathrm{H}$-like (in $62 \%$ of tumor samples) is the most commonly present. We observed its immunoreactivity to be concentrated mostly within tumour stroma and blood vessels (Fig. 1 A, B). CD59 immunoreactivity was detected in 50\% of examined tumors. Its immunoreactivity was present both at the cell membrane and within the cytoplasm of cancer cells, on the medium intensity level. Vascular structures within tumour tissue were also positively stained. Interestingly, in some parts of cancer nests, especially on the border zone between the normal and malignant tissue increased CD59 immunoreactivity was observed (Fig. 1 E, F). CD55 staining was detected only in $4.4 \%$ of examined samples. CD55 was observed predominantly within cancer tubules (Fig. 1C, D). However statistical analysis did not show any significant difference between the examined inhibitors immunoreactivity levels and any of the clinical parameters (age, sex, tumour grade, menopausal status or FIGO stage). 

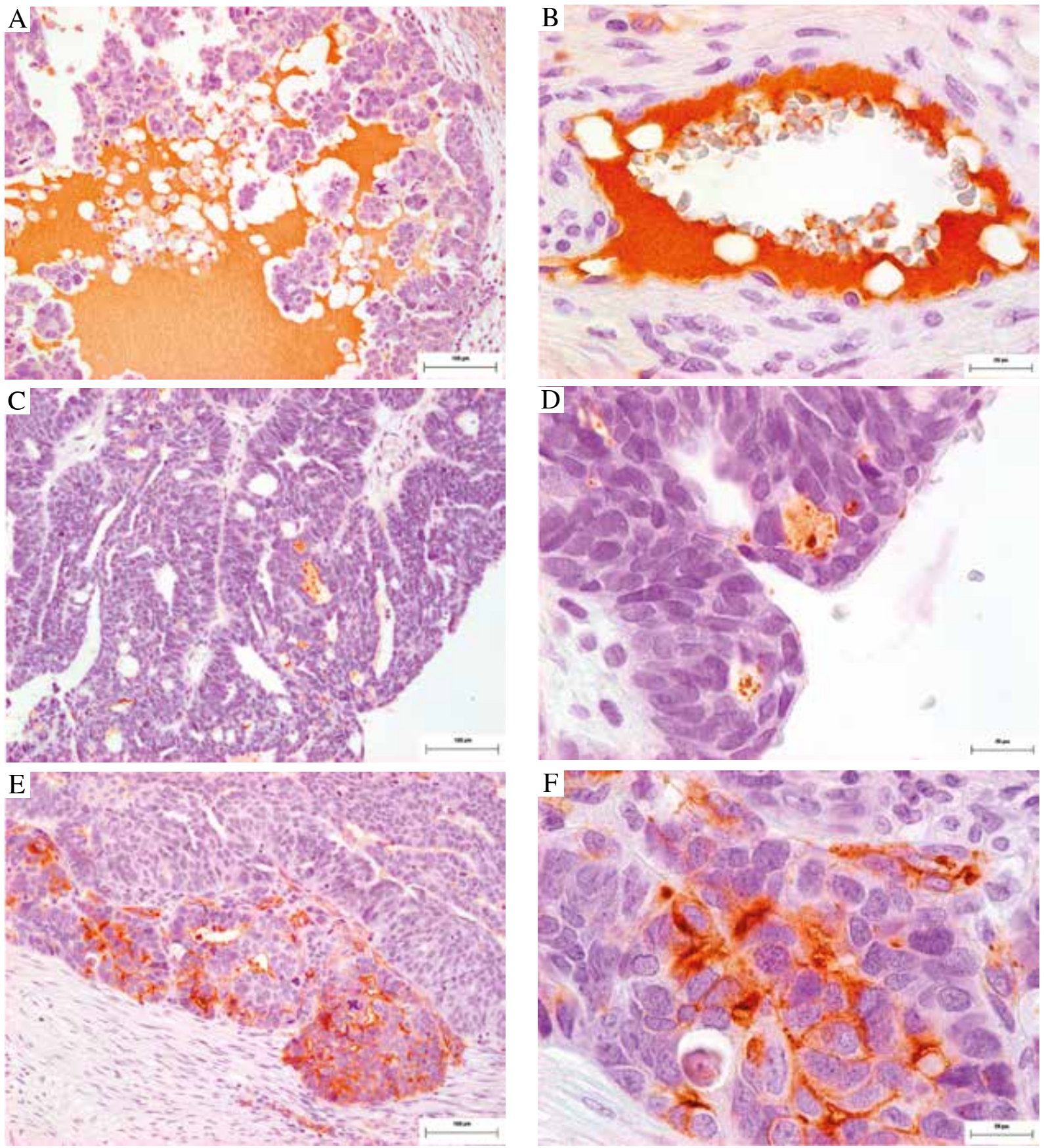

Fig. 1. Factor H/H-like (A, B), CD55 (C, D) and CD59 (E, F) immunoreactivity in the representative image of ovarian cancer tissue; note the prominent factor $\mathrm{H}$ presence within tumor stroma (A) and increased CD59 immunoreactivity on the border zone of the tumour and adjacent normal tissue (E); scale bar A, C, E $100 \mu \mathrm{m}, \mathrm{B}, \mathrm{D}, \mathrm{F} 20 \mu \mathrm{m}$

\section{Discussion}

The ineffective therapy of ovarian and corpus uteri cancers enforces the search of new methods for standard and adjuvant treatment in these indications. The immunotherapy with monoclonal antibodies appeared to be successful in the case of hematological malignancies like i.e.
Rituximab use in non-Hodgkin lymphomas or chronic leukocytic leukemia. Such therapy for solid tumors encounters several difficulties, but attempts to combine the immunotherapy with standard chemotherapy indicate that at least in some percentage of patients it has higher potential of cure with regard to e.g. lung or prostate cancer [19, 20]. Disadvantages of solid tumor therapy may be heteroge- 
neous distribution of targeted antigens, non-uniform blood supply or insufficient penetration of antibodies into the tumor mass. One of the main obstacles to cancer immunotherapy with monoclonal antibodies in solid tumors is the presence of complement inhibitors which limits the efficacy of this therapy. The presence of complement inhibitors was documented in many types of cancer. Although there is no unequivocal message, the majority of work suggests that in many cancer types examined, at least one complement inhibitor is present in the upregulated form.

To date the expression of membranous complement inhibitors in ovarian cancer has not been fully established. Membrane bound complement inhibitors were examined by Bjorge et al., who found a broad expression of CD59 in the examined group and more heterogeneous CD55 immunoreactivity in ovarian cancer tissue [21]. Similar results, underlying the CD59 role in, among others, ovarian cancer cells in vitro in defense against complement attack was shown by Donin et al. [22]. They concluded that blocking of CD59 with specific antibodies enhances complement action on cells in vitro. Similarly promising results with the other way of blocking complement inhibitors were shown by Mamidi et al., where upon blockade of membranous complement inhibitors by siRNA, the prominent downregulation was observed, what subsequently led to complement dependent cytotoxicity augmented by $70 \%$ in established ovarian cancer cell line in vitro [23].

Factor $\mathrm{H}$ and factor $\mathrm{H}-$ like production by ovarian cancer cells was presented in vitro in an established cell line [24]. Results described in this work indicate that ovarian cancer cells produce and actively secrete both forms of the factor $H$ gene product with the prevalence of its truncated form. In addition, the elevated level of factor $\mathrm{H}$ was detected in ascites fluids from patients with this indication. These results are consistent with the data presented in our work, since we observed in our experimental group that the most common among inhibitors examined is the presence of factor $\mathrm{H}$ either bound to the surface of cancer cells or present in the tumour stroma.

The complement inhibitors in corpus uteri cancer were studied by Murray et al. who showed that membrane bound complement inhibitors are upregulated in malignant endometrial tissue [25]. Moreover, as was shown on corpus uteri cancer cell lines, downregulation of CD55 and CD59 sensitizes cancer cells to complement mediated cytotoxicity [26].

The application of immunotherapy with monoclonal antibodies to cancer treatment occurred to be successful in many types of human malignancies. The presence of complement inhibitors localized on the cancer cell surface is mentioned as one of the main reasons for the lack of success in this type of treatment. However, recent growing data testify to the significant role of tumor microenvironment in the cancer cells escape from both standard systemic as well as immunotherapeutic treatment [27-29]. The aims of concurrent therapeutic options are focused not only on destroying cancer cells but also on inducing more widespread response in the local tumor microenvironment. In this context before applying any immunotherapeutic approach to ovarian or corpus uteri cancer, not only a membrane-bound inhibitors blockade, but also the fluid phase such as factor $\mathrm{H}$ present in the local tumor milieu should be considered. In summary, we conclude that essential components of future therapeutic strategies should include combination treatments aimed at dealing with the complement inhibitors, together with rational approaches to patient selection.

This project was financed from the funds of the $\mathrm{Na}$ tional Science Centre granted by decision No. DEC2011/01/D/NZ7/04688.

The authors declare no conflict of interest.

\section{References}

1. Jemal A, Bray F, Center MM, et al. (2011): Global cancer statistics. CA Cancer J Clin 61: 69-90.

2. Robak T (2014): Current and emerging monoclonal antibody treatments for chronic lymphocytic leukemia: state of the art. Expert Rev Hematol: 1-17.

3. Nicholson-Weller A, Burge J, Fearon DT, et al. (1982): Isolation of a human erythrocyte membrane glycoprotein with decay-accelerating activity for $\mathrm{C} 3$ convertases of the complement system. J Immunol 129: 184-189.

4. Medof ME, Walter EI, Rutgers JL, et al. (1987): Identification of the complement decay-accelerating factor (DAF) on epithelium and glandular cells and in body fluids. J Exp Med 165: 848-864.

5. Brodbeck WG, Liu D, Sperry J, et al. (1996): Localization of classical and alternative pathway regulatory activity within the decay-accelerating factor. J Immunol 156: 2528-2533.

6. Li L, Spendlove I, Morgan J, et al. (2001): CD55 is over-expressed in the tumour environment. Br J Cancer 84: 80-86.

7. Durrant LG, Chapman MA, Buckley DJ, et al. (2003): Enhanced expression of the complement regulatory protein CD55 predicts a poor prognosis in colorectal cancer patients. Cancer Immunol Immunother 52: 638-642.

8. Stefanova I, Hilgert I, Kristofova H, et al. (1989): Characterization of a broadly expressed human leucocyte surface antigen MEM-43 anchored in membrane through phosphatidylinositol. Mol Immunol 26: 153-161.

9. Meri S, Morgan BP, Davies A, et al. (1990): Human protectin (CD59), an 18,000-20,000 MW complement lysis restricting factor, inhibits C5b-8 catalysed insertion of C9 into lipid bilayers. Immunology 71: 1-9.

10. Rollins SA, Sims PJ (1990): The complement-inhibitory activity of CD59 resides in its capacity to block incorporation of C9 into membrane C5b-9. J Immunol 144: 3478-3483.

11. Shang Y, Chai N, Gu Y, et al. (2014): Systematic immunohistochemical analysis of the expression of CD46, CD55, and CD59 in colon cancer. Arch Pathol Lab Med 138: 910-919.

12. Kesselring R, Thiel A, Pries R, et al. (2014): The complement receptors CD46, CD55 and CD59 are regulated by the tumour microenvironment of head and neck cancer to facilitate escape of complement attack. Eur J Cancer 50: 2152-2161. 
13. Madjd Z, Pinder SE, Paish C, et al. (2003): Loss of CD59 expression in breast tumours correlates with poor survival. J Pathol 200: 633-639.

14. Pangburn MK, Schreiber RD, Muller-Eberhard HJ (1977): Human complement $\mathrm{C} 3 \mathrm{~b}$ inactivator: isolation, characterization, and demonstration of an absolute requirement for the serum protein beta1 $\mathrm{H}$ for cleavage of $\mathrm{C} 3 \mathrm{~b}$ and $\mathrm{C} 4 \mathrm{~b}$ in solution. J Exp Med 146: 257-270.

15. Pangburn MK (2000): Host recognition and target differentiation by factor $\mathrm{H}$, a regulator of the alternative pathway of complement. Immunopharmacology 49: 149-157.

16. Kristensen T, Tack BF (1986): Murine protein $\mathrm{H}$ is comprised of 20 repeating units, 61 amino acids in length. Proc Natl Acad Sci U S A 83: 3963-3967.

17. Zipfel PF, Skerka C (1999): FHL-1/reconectin: a human complement and immune regulator with cell-adhesive function. Immunol Today 20: 135-140.

18. Junnikkala S, Jokiranta TS, Friese MA, et al. (2000): Exceptional resistance of human $\mathrm{H} 2$ glioblastoma cells to complement-mediated killing by expression and utilization of factor $\mathrm{H}$ and factor $\mathrm{H}$-like protein 1. J Immunol 164: 6075-6081.

19. Mostafa AA, Morris DG (2014): Immunotherapy for Lung Cancer: Has it Finally Arrived? Front Oncol 4: 288.

20. Tse BW, Jovanovic L, Nelson CC, et al. (2014): From Bench to Bedside: Immunotherapy for Prostate Cancer. Biomed Res Int 2014: 981434.

21. Bjorge L, Hakulinen J, Wahlstrom T, et al. (1997): Complement-regulatory proteins in ovarian malignancies. Int J Cancer 70: $14-25$.

22. Donin N, Jurianz K, Ziporen L, et al. (2003): Complement resistance of human carcinoma cells depends on membrane regulatory proteins, protein kinases and sialic acid. Clin Exp Immunol 131: 254-263.

23. Mamidi S, Cinci M, Hasmann M, et al. (2013): Lipoplex mediated silencing of membrane regulators (CD46, CD55 and CD59) enhances complement-dependent anti-tumor activity of trastuzumab and pertuzumab. Mol Oncol 7: 580-594.

24. Junnikkala S, Hakulinen J, Jarva H, et al. (2002): Secretion of soluble complement inhibitors factor $\mathrm{H}$ and factor $\mathrm{H}$-like protein (FHL-1) by ovarian tumour cells. Br J Cancer 87: 1119-1127.

25. Murray KP, Mathure S, Kaul R, et al. (2000): Expression of complement regulatory proteins-CD $35, \mathrm{CD} 46, \mathrm{CD} 55$, and CD 59-in benign and malignant endometrial tissue. Gynecol Oncol 76: 176-182.

26. Bellone S, Roque D, Cocco E, et al. (2012): Downregulation of membrane complement inhibitors CD55 and CD59 by siRNA sensitises uterine serous carcinoma overexpressing Her $2 /$ neu to complement and antibody-dependent cell cytotoxicity in vitro: implications for trastuzumab-based immunotherapy. Br J Cancer 106: 1543-1550.

27. Nelson D, Fisher S, Robinson B (2014): The "Trojan Horse" approach to tumor immunotherapy: targeting the tumor microenvironment. J Immunol Res 2014: 789069.

28. Fang H, Declerck YA (2013): Targeting the tumor microenvironment: from understanding pathways to effective clinical trials. Cancer Res 73: 4965-4977.

29. Lunardi S, Muschel RJ, Brunner TB (2014): The stromal compartments in pancreatic cancer: are there any therapeutic targets? Cancer Lett 343: 147-155. 